\title{
Stem Cells and Regeneration in Plants
}

\author{
Giovanni Sena \\ Department of Life Sciences, South Kensington Campus, Imperial College London, London, UK
}

\section{Key Words}

Plants · Roots · Meristem · Plant stem cells $\cdot$ Root

regeneration · Organogenesis · Developmental plasticity

\begin{abstract}
Background: Plants are characterized by indeterminate post-embryonic development that is evident, for example, in the continuous branching of shoots and roots. High competence to regenerate tissues is another consequence of such intrinsic developmental plasticity in plants. It has been suggested that specialized groups of cells within plant meristems should be compared to stem cells in animals, but the utility of this label in the context of post-embryonic plant development and regeneration is often debated. Summary: This paper is organized into 3 short sections, where (a) key observations and experimental results on tissue regeneration in plants - mainly in the model system Arabidopsis thaliana, (b) stem cell activity and (c) their role in regeneration are described. The main focus is maintained on the critical aspects of defining stem cell-ness in plants, particularly in the context of tissue regeneration. A number of recent excellent reviews are cited throughout the text to give the reader the appropriate tools to dig deeper into the various stimulating topics introduced here. Key Messages: Despite the remarkable somatic developmental plasticity characterizing post-embryonic development in plants, use of the classic
\end{abstract}

concept of stem cells has been imported from the animal literature with the goal of facilitating our understanding and description of plant developmental processes. It is not clear if this is the case, especially in light of the recent experimental results on root regeneration in Arabidopsis mutants.

(c) 2014 S. Karger AG, Basel

\section{Introduction}

Even though multi-cellularity is likely to have evolved independently in plants and animals, some developmental processes seem to be present in both kingdoms. Regeneration is one of them. In fact, the regeneration of body parts - such as roots, shoots or leaves - is a common feature in plant life and can be studied to explore the intrinsic constraints within developmental biology.

Various examples of regeneration in animals are linked to the activity of adult stem cells [1]. Is it the same in plants? The modern concept of a stem cell, originally emerging in the context of tissue homeostasis in animals [2], has been imported to the field of plant development, triggering some debate [3]. The exact role of stem cells in the mechanism of tissue regeneration in plants remains generally unclear. In this brief paper I highlight the main points currently under discussion regarding the meaning of stem cells within plant development and regeneration.

\section{KARGER}

E-Mail karger@karger.com

www.karger.com/nee
(C) 2014 S. Karger AG, Basel

$1660-2129 / 14 / 1262-0035 \$ 39.50 / 0$
Giovanni Sena

Department of Life Sciences, South Kensington Campus

Imperial College London

London SW7 2AZ (UK)

E-Mail g.sena@imperial.ac.uk 

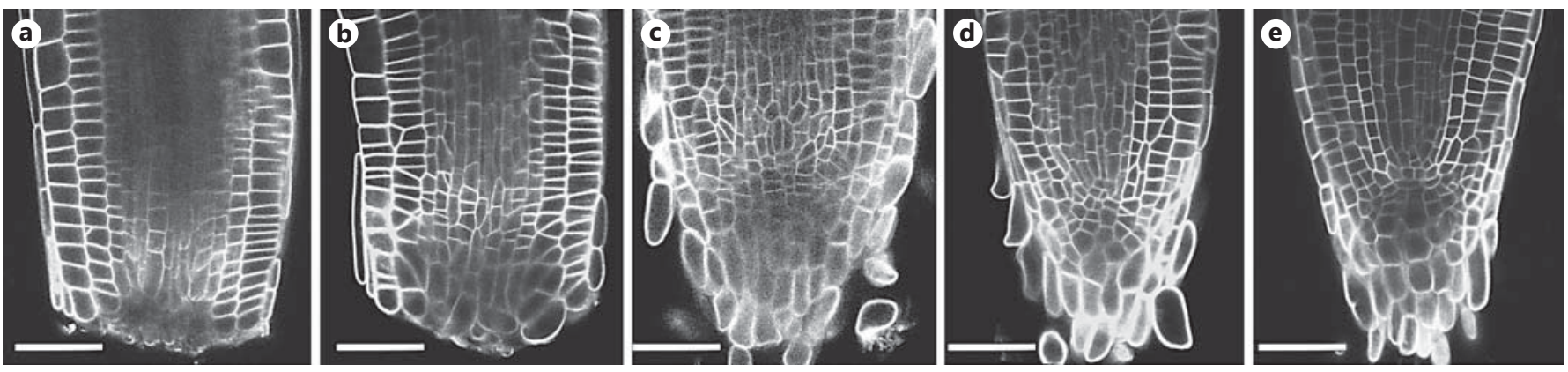

Fig. 1. Time lapse series of the regeneration of a single root tip in A. thaliana after physical excision. Transgenic line expressing yellow fluorescent protein in the plasma membrane (WAVE-131, from N. Geldner). Images were generated by R. Elsmore in the laboratory of G. Sena at 5 (a), 24 (b), 48 (c), 72 (d), and 96 hours (e) after excision. Scale bars $=50 \mu \mathrm{m}$.

\section{Regeneration in Plants}

The term regeneration is used to describe a wide spectrum of phenomena [4]. A first distinction can be made between reparative and restorative regeneration, where the former refers to the repair of local wounding in single tissues and the latter indicates the reconstruction of a whole structure (composed of multiple tissues), organ or even individual. Plants are capable of both kinds of regeneration, but in this paper I will focus on restorative regeneration and its relation to adult stem cells.

One crucial aspect of plant life is post-embryonic development. A plant embryo is just a stripped-down version of the adult individual, typically with just 1 or $2 \mathrm{em}$ bryonic leaves (cotyledons) and 1 embryonic root. After germination, a plant will develop complex shoot and root structures, with numerous lateral shoots and roots, leaves and sometimes flowers. Crucially, the number of each of these appendices is variable throughout the life of an individual and cannot be guessed by looking at the embryo. This is due to the remarkable plasticity of post-embryonic development in plants, which sustains the ability to (re)generate whole new organs such as roots, leaves or flowers, during adult life.

The ectopic appearance of completely new organs is generally called de novo organogenesis and can be easily observed in living plants (in situ) as well as artificially induced in tissue cultures in the laboratory (in vitro). In vitro regeneration became possible thanks to the seminal work of Skoog and Miller [5], who showed how to obtain a proliferating mass of cells (callus) from plant extracts and subsequently induce the ectopic development of roots or shoots by simply varying the ratio of two impor- tant phytohormones (cytokinin and auxin) in the culture medium. This seems to hold true also for the extreme case where an entire plant can be regenerated from a single somatic cell [6]. A second class of regenerative processes in plant development is the very peculiar somatic embryogenesis, where true embryos are formed from somatic cells either in situ or in vitro [7]. Finally, plants exhibit proper in situ organ regeneration, such as the restoration of completely excised root tips (fig. 1) or leaf margins, in some aspects reminiscent of limb regeneration in metazoans [8].

\section{Stem Cells in Plants}

The modern definition of stem cells adopted for animal tissues calls for a population of cells that are: (a) undifferentiated (i.e. without any molecular or morphological marker associated with cellular differentiation), (b) able to maintain the size of the population (self-renewal) through controlled proliferation, and (c) able to generate various cell types [9]. Properties (b) and (c) imply some form of developmentally asymmetric cell division as well as multipotency. Interestingly, it has been observed that the asymmetry of a division, giving rise to both undifferentiated stem cells and cells committed to mature fates, could manifest itself either deterministically at each single mitotic event or statistically at the population level [10]. Animal stem cells are typically embedded in a cellular environment - called the stem cell niche - that contributes to keeping them in an undifferentiated state and capable of asymmetric divisions [11]. DOI: $10.1159 / 000360658$ 
A system optimized to maintain a relatively small pool of stem cells and at the same time sustain fast growth through a high proliferation rate might exhibit a second class of cells - termed transit cells - which act downstream of stem cells to amplify the portion of their progeny that is committed to differentiation [9].

Plant continuous post-embryonic development requires the existence of an inexhaustible source of new cells. Apical meristems are the specialized regions at the apex of shoots and roots where continuous cell proliferation sustains growth throughout the lifetime of the individual [12]. Shoot and root branching, both manifestations of post-embryonic organogenesis, are dependent on the generation of new (lateral) meristems. Even though the histories of the two terms - stem cell and meristem are separate [2], it is tempting to draw a parallel between meristematic cells in plants and adult stem cells in animals. In fact, it can be argued that the described concepts of stem cell, stem cell niche, and transit cell could be matched by specific cell populations within plant meristems [13]. While a complete review of this topic is clearly beyond the scope of this brief paper, the wide attention attracted by the concepts of stem cells and their niches in plant development can be deduced by recent excellent reviews and references therein [14]. It should be noted that, despite the growing body of work taking such labels for granted, the utility of adopting them in the context of plant development could still be debated today as it was 10 years ago [3].

Let us touch on some of the disputed points associated with the idea of stem cells in plants. It is generally understood that adult stem cells in metazoans are developed during embryogenesis and maintained during the lifetime of the individual as subpopulations segregated from the rest of differentiating somatic cells. In first approximation this seems to hold true in animals both for germline stem cells and adult somatic stem cells. It has been suggested that one potential advantage of this strategy is the ability to hold on to a pool of relatively undifferentiated cells which have gone through a limited number of mitotic events and potentially a minimal accumulation of DNA mutations [9]. Interestingly, this idea of a segregated cell population does not apply to plants, where differentiated somatic cells can generate new adult somatic stem cells, for example, during shoot and root branching and even germlines as during flowering. Therefore, if a stem cell niche may be considered in animals a temporal capsule for embryonic genetic state, or a reservoir of the original zygotic genome, in plants each lateral meristem should be considered a new temporal capsule by itself, potentially containing a number of DNA mutations locally accumulated until the branching event. This seems to dilute one of the supposed advantages of including stem cells in a multicellular body.

A second interesting point is related to the peculiar plasticity of stem cells in an adult body. In plants, many somatic cell types can divide and replace neighbouring dead cells, including stem cells themselves, as shown for example in spectacular laser ablation experiments in the root meristem of Arabidopsis [15]. Somatic differentiated cells can even regenerate an entire stem cell niche when excised, as shown, for example, in the regeneration of the whole root apex in Arabidopsis [16]. What does this say about the distribution of cellular multipotency in an adult plant? If this is not a feature restricted to a localized subpopulation of cells anymore, the definition of stem cell seems weakened, possibly less useful, and maybe even confusing.

Finally, can we identify a stem cell transcriptional state in plants? This is an important question which goes to the heart of stem cell-ness in the context of developmental biology. It is a point that has been experimentally addressed both in root [17] and in shoot [18] apical meristems. Even though none of these results seems conclusive when considered one at the time, the search for a transcriptional network defining stem cell niches in plants can be considered to be just in its early stages.

Taken together, the results described above raise the legitimate question of whether it is actually helpful to introduce the special category of stem cells for plants. Recent renewed efforts to draw even closer parallels between the cases of stem cell-ness in both animal and plant kingdoms will certainly once again stir up the debate [19].

\section{The Role of Stem Cells in Plant Organ Regeneration}

Most of the data currently available on the link between plant stem cells and organ regeneration comes from experiments on the model system Arabidopsis thaliana. Following the definitions indicated above, here I will focus on the case studies of in vitro organogenesis and in situ root regeneration. The first case refers to the possibility of regenerating plant organs by culturing explants and inducing callus, a tissue for a long time considered to be a quite disorganized mass of undifferentiated cells [20]. In fact, it has been recently shown that callus - whether derived from root or shoot explants - develops morphological and transcriptional analogies with root tissues [21]. Interestingly, mutants unable to produce the initial 
cell divisions required for the formation of a stem cell niche in a new lateral root will also show a significant reduction of callus-induced shoot and root in in vitro organogenesis [21]. These and further supporting results reviewed in detail in recent publications $[8,22]$ suggest that in vitro organogenesis from callus recapitulates the developmental program of lateral root formation. Although the late generation of a shoot stem cell niche is required for shoot organogenesis from callus [23], and although transcriptional analyses of in vitro organ regeneration from callus have been carried out [24,25], it is still unclear whether a proper root stem cell niche is in fact required early on in this process.

As already mentioned, a number of experiments in different plant species have shown that root tips can regenerate when decapitated [8]. This is also true in Arabidopsis, where a completely new root apex will form within a few days with the same general proportions and internal organization of the uncut one [16]. If an excision is performed to completely remove the root apical stem cell niche, this will simply reappear in the newly regenerated tissue through proliferation and repatterning of the stump made of differentiated somatic cells [16]. This appears more like the self-organization of a rather mature tissue rather than the self-renewal of an undifferentiated niche. On a smaller scale, the same phenomenon is observed following the already mentioned laser-assisted in situ wounding of the niche [15].

Surprisingly, root apex regeneration occurs even in mutants where the apical stem cell niche fails early on in post-embryonic development, leading to stem cell differentiation, perturbation of the internal pattern and eventually cessation of root growth [16]. In these experiments, a mutant root is genetically unable to maintain viable stem cells but will still regenerate a root apex - al- beit with a mutant phenotype and incapacity for continuous growth.

These results seem to separate continuous post-embryonic growth, which requires functional stem cells, from tissue regeneration per se, which at least in this example does not depend on functional stem cells. Instead, regeneration in either wild-type cells or mutants does not happen if the root is decapitated in a way that completely eliminates the proximal transit cells as well [16], indicating that this population of proliferating but differentiating cells is necessary for the process.

\section{Conclusions}

A label can be quite useful for capturing the essence of a complex concept, and it becomes desirable if it simplifies the description of a wider process. Is this the case for the popular stem cell tag in the context of organ regeneration in plants? Here, I tried to highlight the remarkable plasticity found in plant post-embryonic development, where the idealistic uniqueness of a self-renewing population of multipotent cells appears thinned. The main question remains whether a precise definition of stem cell helps to clarify the phenomenon of regeneration in plants and, more in general, their post-embryonic development.

From a more biological - and less semantic - point of view, it appears as if ageing adult metazoans required small pools of young cells capable of repairing and regenerating tissues otherwise incapable of post-embryonic development, while plants found in their intrinsic developmental plasticity the source of unlimited potential tissue regeneration.

Should we not say that plants, in fact, continuously regenerate themselves as they grow?

\section{References}

1 Sanchez Alvarado A: Regeneration in the metazoans: why does it happen? Bioessays 2000;22:578-590.

-2 Ramalho-Santos M, Willenbring $\mathrm{H}$ : On the origin of the term 'stem cell'. Cell Stem Cell 2007; 1:35-38.

3 Laux T: The stem cell concept in plants: a matter of debate. Cell 2003;113:281-283.

$\checkmark 4$ Dinsmore CE, American Society of Zoologists: A History of Regeneration Research: Milestones in the Evolution of a Science. Cambridge, Cambridge University Press, 1991.

5 Skoog F, Miller CO: Chemical regulation of growth and organ formation in plant tissues cultured in vitro. Symp Soc Exp Biol 1957;54: 118-130.

6 Steward FC: Growth and organized development of cultured cells. 3. Interpretations of the growth from free cell to carrot plant. Am J Bot 1958;45:709-713.

7 Elhiti M, Stasolla C, Wang A: Molecular regulation of plant somatic embryogenesis. In $\mathrm{Vi}$ tro Cell Dev Biol Plant 2013;49:631-642.

$>8$ Sugimoto K, Gordon SP, Meyerowitz EM: Regeneration in plants and animals: dedifferentiation, transdifferentiation, or just differentiation? Trends Cell Biol 2011;21:212218 .
9 Potten CS, Loeffler M: Stem cells: attributes, cycles, spirals, pitfalls and uncertainties: lessons for and from the crypt. Development 1990;110:1001-1020.

10 Klein AM, Simons BD: Universal patterns of stem cell fate in cycling adult tissues. Development 2011;138:3103-3111.

11 Schofield R: The relationship between the spleen colony-forming cell and the haemopoietic stem cell. Blood Cells 1978;4:7-25.

12 Machida Y, Fukaki H, Araki T: Plant meristems and organogenesis: the new era of plant developmental research. Plant Cell Physiol 2013;54:295-301. 
13 Ivanov VB: Meristem as a self-renewing system: maintenance and cessation of cell proliferation (a review). Russ J Plant Physiol 2004; 51:834-847.

14 Aichinger E, Kornet N, Friedrich T, Laux T: Plant stem cell niches. Annu Rev Plant Biol 2012;63:615-636.

15 Xu J, Hoflhuis H, Heidtsra R, Sauer M, Friml J, Scheres B: A molecular framework for plant regeneration. Science 2006;311:385-388.

16 Sena G, Wang XN, Liu HY, Hofhuis H, Birnbaum KD: Organ regeneration does not require a functional stem cell niche in plants. Nature 2009;457:1150-1153.

17 Nawy T, Lee JY, Colinas J, Wang JY, Thongrod SC, Malamy JE, et al: Transcriptional profile of the Arabidopsis root quiescent center. Plant Cell 2005;17:1908-1925.
18 Yadav RK, Girke T, Pasala S, Xie M, Reddy GV: Gene expression map of the Arabidopsis shoot apical meristem stem cell niche. Proc Natl Acad Sci USA 2009;106:4941-4946.

19 Somorjai IML, Lohmann JU, Holstein TW, Zhao Z: Stem cells: a view from the roots. Biotechnol J 2012;7:704-722.

20 Smith R: Plant Tissue Culture: Techniques and Experiments. Waltham, Academic Press, 2012.

21 Sugimoto K, Jiao Y, Meyerowitz EM: Arabidopsis regeneration from multiple tissues occurs via a root development pathway. Dev Cell 2010;18:463-471.

22 Van Norman JM, Xuan W, Beeckman T, Benfey PN: To branch or not to branch: the role of pre-patterning in lateral root formation. Development 2013;140:4301-4310.
23 Gordon SP, Heisler MG, Reddy GV, Ohno C, Das P, Meyerowitz EM: Pattern formation during de novo assembly of the Arabidopsis shoot meristem. Development 2007;134: 3539-3548.

24 Che P, Lall S, Nettleton D, Howell SH: Gene expression programs during shoot, root, and callus development in Arabidopsis tissue culture. Plant Physiol 2006;141:620-637.

25 Chatfield SP, Capron R, Severino A, Penttila PA, Alfred S, Nahal H, et al: Incipient stem cell niche conversion in tissue culture: using a systems approach to probe early events in WUSCHEL-dependent conversion of lateral root primordia into shoot meristems. Plant J 2013;73:798-813. 